\title{
Tendencias actuales en las tradicionales herramientas de cooperación internacional en Ciencia y Tecnología. El caso de la Unión Europea y la República Argentina
}

Marta Cabeza*

\section{Resumen}

Este artículo aborda la cooperación internacional en Ciencia y Tecnología entre la República Argentina y la Unión Europea (UE). Se contextualiza este objeto de estudio en ámbitos más amplios, como son la cooperación internacional norte-sur y el birregionalismo, para luego recorrer los períodos de gobierno de Cristina Fernández de Kirchner y de Mauricio Macri. Se finaliza con el análisis del inicio del gobierno de Alberto Fernández para focalizar las principales tendencias que se observan en el actual contexto de pandemia.

En este recorrido se identifica como principal falencia de esta cooperación la unidireccionalidad, tanto en el diseño como en la aplicación y en la evaluación de las políticas, desde la UE hacia terceros países. Esta modalidad no aportaría al desarrollo de las potencialidades de los sistemas productivos locales y tendría pocas consecuencias positivas sobre la población argentina en su conjunto.

Palabras claves: cooperación en Ciencia y Tecnología - Argentina - Unión Europea - cooperación internacional

\section{Current trends in traditional international cooperation tools in Science and Technology. The case of the European Union and Argentina}

\begin{abstract}
This article analyzes the international cooperation in Science and Technology between the Argentine Republic and the European Union (EU). This object of study is contextualized in wider areas, such as international north-south cooperation and bi-regionalism, to then go through the periods of government of Cristina Fernández de Kirchner and Mauricio Macri. It ends with the analysis of the beginning of the Alberto Fernández government to focus on the main trends observed in the pandemic current context.

In this approach, unidirectionality is identified as the main flaw in this cooperation, both in the design and in the application and in the evaluation of policies, from the EU to third countries. This modality would not contribute to the development of the potential of local productive systems and would have few positive consequences on the Argentine population as a whole.
\end{abstract}

Key Word: Science and Technology cooperation - Argentina - European Union - international cooperation

TRABAJO RECIBIDO: 20/04/2021

TRABAJO ACEPTADO: 04/06/2021

Esta obra está bajo una licencia internacional https://creativecommons.org/licenses/by-sa/4.0/

\footnotetext{
* Licenciada en Relaciones Internacionales, Facultad de Ciencia Política y Relaciones Internacionales, Universidad Nacional de Rosario (UNR, Argentina). Magíster en Integración y Cooperación Internacional, Universidad Nacional de Rosario (CERIR-CEI-UNR, Argentina). Correo electrónico: marcabeza@yahoo.com
} 


\section{Introducción}

Este trabajo tiene como objetivo general el abordaje de la cooperación internacional en Ciencia y Tecnología (CyT) entre la República Argentina y la Unión Europea (UE) tomando indicadores en varios niveles: por un lado, los tratados bilaterales firmados por Argentina con la región, llamado también nivel formal; por otro, la participación del país sudamericano en los Programas Marco (PM) europeos, situados en un nivel instrumental; y, por último, apoyándose en una visión amplia de la cooperación internacional en CyT, este análisis también toma en cuenta muchas actividades y prácticas como el intercambio de investigadores y becarios, el desarrollo de programas conjuntos de formación, el desarrollo de productos para la industria, la organización de seminarios, entre otras.

En cuanto al recorte temporal, el inicio está dado por el alcance del "Consenso del Milenio" en el año 2000, con el que se establecen objetivos e instrumentos para la construcción de una nueva arquitectura de la cooperación internacional. Los elementos novedosos de este enfoque residen en la necesidad de combinar la cooperación norte-sur, sur-sur y triangular y, dentro en esos variados esquemas, el desempeño de actores y agentes en múltiples niveles. Inserta en este nuevo contexto internacional es que se analiza la cooperación en CyT entre Argentina y la UE, desde el período de gobierno de Cristina Fernández de Kirchner, quien asume en diciembre del 2007, llegando hasta la identificación de las tendencias actuales y los cambios que podría provocar la pandemia de Covid-19 en el área.

Las fuentes consultadas para la recolección de datos fueron las páginas webs oficiales del Ministerio de Ciencia, Tecnología e Innovación (conocido por su sigla abreviada MINCYT), del Ministerio de Relaciones Exteriores, Comercio Internacional y Culto (MRECIC), de la UE y de la embajada argentina ante este organismo; los informes académicos de otros investigadores y las notas de prensa de medios argentinos y europeos.

Como punto de partida, es preciso reflexionar sobre la necesidad de analizar esta cooperación en un marco de comprensión integral más amplio y complejo, que incluye la totalidad de la política en CyT de la Argentina y, dentro de ésta, la totalidad de la cooperación internacional en CyT. Esto nos permitirá comprender mejor qué objetivos o puntos nodales se plantea el país en la interacción con la UE, participando de programas y proyectos, pudiendo evaluar así la utilidad o el aporte real sobre las capacidades nacionales de producción de conocimiento, innovación y avance técnico. Es decir, que la cooperación en CyT con la UE constituye un sector parcial en un marco más amplio de la cooperación internacional y de la política nacional del país en CyT (Demarchi, 2020; Kern, 2008). Por esto es que, a medida que se caracteriza la cooperación con la UE, se vincula con las decisiones nacionales en el área, intentado valorar la modalidad del trabajo de cooperación, en un necesario nexo entre ésta y los objetivos de la Política Exterior del Estado.

En cuanto a los actores, y dada la complejidad de los vínculos internos y externos, se amplía el concepto clásico que otorgaba el protagonismo a los Ministerios de Relaciones Exteriores y se hace necesario habilitar nuevos roles hacia el interior de los Estados. Es por esto que se incluye también en el análisis el accionar del Ministerio de Ciencia, Técnica e Innovación ${ }^{1}$ junto con las Comisiones de Ciencia y Tecnología que desempeñan su labor en el Congreso de la Nación, y también se tienen en cuenta las políticas vinculadas al Consejo Nacional de Investigaciones Científicas y Técnicas (CONICET).

\section{Los encuadres más amplios: la Agenda 2030 y el birregionalismo}

Para iniciar, resulta útil vincular la problemática de la cooperación en CyT entre Argentina y la UE, incorporada en los ámbitos que la contienen y que se mencionaron en la

\footnotetext{
${ }^{1}$ Luego Secretaría de Ciencia y Técnica con el gobierno de Mauricio Macri, para volver a recuperar el nivel de Ministerio con el gobierno de Alberto Fernández.
} 
introducción, con una perspectiva conceptual y teórica más amplia que nos permita entender mejor el fenómeno.

Un primer encuadre para entender el contexto amplio de esta cooperación refiere al gran marco de cooperación internacional entre el norte y el sur: con el "Consenso 2000" o "Cumbre del Milenio" 189 países se reunieron en la Asamblea General de Naciones Unidas para planificar cómo unir esfuerzos y voluntades para renovar la cooperación internacional en pos de combatir el hambre y la pobreza en el mundo. Unos meses más tarde el Secretario General propuso los Objetivos de Desarrollo del Milenio (ODM), que establecían el año 2015 como fecha de cumplimiento (Sanahuja y Tezanos Vázquez, 2017).

Pero cuando se finaliza la implementación de los ODM, en el 2015, se observa que los resultados no habían sido tan exitosos como los esperados. Las graves desigualdades entre los países desarrollados y los países en vías de desarrollo seguían existiendo. Es por ello que se decide por unanimidad seguir trabajando en una agenda más amplia que incluyera, además, las nuevas problemáticas surgidas en esos años.

Es así que, durante la Conferencia de Financiación para el Desarrollo, en Addis Abeba en el 2015, y a partir de una propuesta desarrollada en la Conferencia de Naciones Unidas sobre el Desarrollo Sostenible (Río +20), de Río de Janeiro en junio de 2012, se propone la nueva Agenda para el Desarrollo Sostenible, llamada también "Agenda 2030". Este documento propone 17 Objetivos de Desarrollo Sostenible (ODS), a su vez detallados en 169 metas, que abarcan distintas temáticas.

Desde el sur se desarrollan fuertes críticas a este esquema de relacionamiento acusado de representar "el intento del viejo 'Occidente' de mantener su liderazgo en la agenda global, apoyándose en la arquitectura institucional y financiera del régimen de ayuda que se fue cristalizando durante el período de la posguerra" (Caria, 2020, p. 265).

Si se considera al conjunto de ayudas y prácticas de cooperación internacional como una institución internacional, se puede coincidir con Caria en que "las agendas de cooperación internacional, en su función de establecer objetivos, metas y estrategias para alcanzarlas, han hecho una contribución significativa a la configuración del orden internacional en las últimas décadas, de modo que se convierten en uno de los terrenos en los que se definen las características de transición hegemónica" (Caria, 2020, p. 267). En este ámbito se favorece la creencia en un imaginario de prosperidad, donde las democracias occidentales marcarían el camino a seguir, aplicando el concepto de desarrollo para un afianzamiento no sólo de la hegemonía norteamericana sino también del predominio de los países europeos. Con las actuales crisis de las democracias occidentales y el surgimiento de nuevos liderazgos internacionales, aunque no terminen de tomar forma, todo este esquema de ideas se pone en tela de juicio. El poder norteamericano y europeo viene siendo discutido desde diferentes alternativas como populismos (Müller, 2017), nacionalismos y hasta sectores de las propias sociedades civiles (Sanahuja, 2012).

En la actualidad, en forma simultánea a estos cambios, las relaciones desiguales de poder a escala global siguen representando el mayor desafío para la "Agenda 2030" (Denk, 2016, p. 49), con prácticas que tienen lugar en un escenario donde la lógica sigue siendo el mercado y el consenso pos Washington.

De esta manera, los ODS pueden ser considerados una iniciativa internacional que se sustenta en discutibles y cuestionadas políticas neoliberales, alejándose de objetivos de desarrollo socialmente más justos y ecológicamente más sostenibles. Además, se cuestiona el carácter voluntario de esta agenda: pese a abordar derechos humanos fundamentales no fue posible otorgar a los ODS el estatus legalmente vinculante que se asegura a los acuerdos multilaterales sobre este ámbito, debido a la falta de consenso entre los países signatarios sobre este aspecto (Caria, 2020; Denk, 2016).

En este marco de cooperación internacional entre el norte y el sur, otra cuestión importante a tener en cuenta es la clasificación de los países según la renta con la consiguiente 
tipología de Países de Renta Media (PRM)², entre los que se encuentra la República Argentina. Agrupar a los países según su renta, sin visibilizar las grandes diferencias que hay entre ellos y ocultado problemáticas hacia el interior de cada nación (Pattacini, 2013), vinculadas entre otras variables con la distribución de esa renta, no es la mejor forma de optimizar la cooperación internacional. Sin embargo, entre las recomendaciones para seleccionar a los países que recibirán ayuda recurrentemente se menciona que sean de renta baja, estableciendo de esta manera que aquellos países que registren ingresos per cápita medianos o mediano-altos no serían potenciales candidatos para recibir Ayuda Oficial al Desarrollo (AOD).

De todos modos, el Consenso Europeo para el Desarrollo ${ }^{3}$ indica que si bien la prioridad de la AOD es "el apoyo a los países menos adelantados (PMA) y otros países de renta baja (PRB), se dejan abiertas perspectivas de enorme relevancia para la mayoría de los países de América Latina y el Caribe, ALC. El apoyo a los países de renta media sigue siendo igualmente importante para alcanzar los ODM. Muchos países de renta media-baja se enfrentan al mismo tipo de dificultades que los de renta baja" (Morazán, Sanahuja y Ayllón, 2011, p.26). Lo cierto es que esta clasificación que tiene en cuenta la renta, y que preocupó mucho a la región latinoamericana, no repercutió del mismo modo en todas las áreas de cooperación internacional. "Los PRM han sido capaces de obtener importantes recursos a través de los cuales avanzaron hacia el fortalecimiento de políticas públicas en temáticas como la ciencia y tecnología" (Demarchi, 2018, p. 9).

Un segundo encuadre de la perspectiva desde dónde podemos analizar la cooperación en CyT entre Argentina y la UE es el interregionalismo: la "asociación estratégica" -como se denominó a la relación entre los países del ALC y la UE- con su primera cumbre celebrada en Río de Janeiro en 1999. Aunque Grabendorff (2014, p. 175) sostiene que esta asociación estratégica se mostró desde el principio muy poco preparada para las profundas trasformaciones internacionales que estaban por venir.

La UE es el principal dador de cooperación en América Latina, con un nutrido sistema de ayuda. Esta densa red de acuerdos y programas de cooperación atravesó lo que puede ser considerada como la "edad de oro" en el período 1990-2015, pero no se mostró lo suficientemente flexible como para acomodarse a los "acelerados cambios en las percepciones mutuas" (Grabendorff, 2014, p. 178) de las primeras décadas del siglo XXI. En el 2010, y con la intención de generar un solo interlocutor y una voz en común en ALC, se crea la Comunidad de Estados Latinoamericanos Caribeños (CELAC), instituida definitivamente en Caracas en el 2011.

En la estructura institucional birregional cada dos o tres años se realiza una cumbre entre la UE y la CELAC. El 8 de enero de 2020 México asumió la presidencia de la CELAC, con una amplia agenda presentada en un acto realizado en Ciudad de México. Como resultado de ello, se han celebrado conversaciones para dar un nuevo impulso a las relaciones entre la UE y ALC. De cualquier manera, el contexto regional y el calendario político en 2020, afectados fuertemente por la pandemia de Covid-19, presentan una serie de obstáculos para la celebración de una cumbre UE-CELAC en un futuro próximo, aunque se sigan explorando las posibilidades de alcanzar un compromiso político de alto nivel.

Mientras tanto, continúa "la cooperación sectorial sobre determinados retos compartidos, como la seguridad ciudadana, las drogas y la investigación y la innovación" (Parlamento Europeo, 2020).

Es pertinente preguntarse si esta dimensión birregional, con sus debilidades, permite algún tipo de intervención de parte de ALC en el diseño de estrategias para la cooperación. El

\footnotetext{
${ }^{2}$ Los PRM son definidos por el Banco Mundial (BM) y la OCDE según el nivel de ingresos (Producto Bruto Interno -PBI- per cápita) de cada economía.

${ }^{3}$ El Consenso Europeo para el Desarrollo establece un nuevo conjunto de principios para la cooperación al desarrollo como parte de la respuesta de la UE a la Agenda 2030 para el Desarrollo Sostenible. Se plasma en la declaración conjunta aprobada el 20 de diciembre del 2005 por las tres principales instituciones de la UE: el Parlamento, el Consejo y la Comisión, junto a los Estados miembros.
} 
informe externo solicitado por el Parlamento Europeo en el 2011 (Morazán, Sanahuja y Ayllón, 2011) menciona el "dilema" que se genera entre el Instrumento de Cooperación al Desarrollo (ICD), que define áreas geográficas y objetivos específicos en función del objetivo general de la cooperación europea y las demandas planteadas en las cumbres birregionales UE-ALC. Este informe sostiene que "no pocas veces el proceso de las cumbres cuestiona las políticas ya antes formuladas por los instrumentos comunitarios" (Morazán, Sanahuja y Ayllón, 2011, p. 7).

Los autores del informe proponen reforzar, dentro de la asociación birregional, la importancia de los Documentos de Estrategia de País (DEP) y los Documentos de Estrategia Regional (DER), como instrumentos para la cooperación internacional ya que estas instancias tienen la característica de ser elaboradas mediante un proceso de diálogo con los Gobiernos nacionales de cada país receptor y con otros actores relevantes. En ellos se definen los objetivos específicos y los sectores de intervención de la cooperación de la UE, si bien se señala que hasta ahora no han sido socializados suficientemente, de tal manera que incluyan también exigencias de la sociedad civil (Morazán, Sanahuja y Ayllón, 2011, p. 8).

Poniendo en diálogo ambos encuadres, la Agenda 2030 y el interregionalismo, con la problemática de la cooperación en CyT de la UE con América Latina, Feld y Kreimer dan cuenta de la "tensión interpretativa" entre las críticas y los análisis más positivos. Existen dos lecturas sobre esta cooperación: un primer grupo que se sitúa en una posición crítica, hablando de sometimiento y subordinación, y otro grupo que aporta una mirada más optimista y rescata las posibilidades de cosmopolitismo e inserción en la ciencia internacional que este tipo de cooperación ofrece (Feld y Kreimer, 2019, p. 782). Sobre estas cuestiones se volverá más adelante.

\section{Modalidades de la cooperación internacional en CyT entre la Argentina y la UE y sus valoraciones críticas}

Entre los indicadores del nivel formal mencionados en la introducción en la cooperación científico técnica entre Argentina y la UE, que marcan hitos o momentos trascendentales en la vinculación, se pueden señalar:

- El Acuerdo de Cooperación Científica y Tecnológica firmado con la UE en 1999, en vigor desde 2001, con el objetivo de incentivar las actividades de colaboración en las áreas de interés común, e impulsar la utilización de los resultados obtenidos para el beneficio económico y social de Argentina y los países miembros de la UE.

- La creación de ABEST, la oficina de enlace entre Argentina y la UE, establecida en 2005, que y que también se orienta a la consolidación de las relaciones entre ambas partes. La intermediación de esta oficina de enlace fue determinante para la creciente participación de instituciones argentinas en la cooperación con la UE.

Cabe mencionar que los países que hoy forman parte de la UE fueron los primeros que firmaron tratados en CyT con Argentina y la región latinoamericana, en los años sesenta, pero es durante las décadas del ochenta y noventa cuando se sellaron la mayor cantidad de acuerdos. Por otra parte, la UE supera a las otras regiones del mundo en términos de cantidad de tratados bilaterales de CyT firmados con nuestro país (Miguel y Ugartermendía, 2010, p. 7). Que la UE sea la región con mayor presencia en la cooperación con Argentina

se explica razonablemente porque fueron éstos los países que impulsaron las políticas de cooperación en CyT en los organismos multilaterales en los años sesenta, y fueron los que iniciaron la firma de tratados bilaterales en el marco de las políticas de 'ayuda al desarrollo'. Esto fue parte de una política general hacia las regiones poco desarrolladas que impulsaron Francia, el Reino Unido, los Países Bajos, Bélgica, Alemania, Portugal y España (junto a Estados Unidos, y más tarde Japón) (Miguel y Ugartermendía, 2010, p. 12). 
Revisando los antecedentes de la cooperación entre América Latina y los países centrales, se advierte que este esquema de cooperación no consideraba, en la gran mayoría de los casos, las necesidades de los países del sur ni los efectos de la cooperación sobre su desarrollo económico sino que la lógica que los movilizaba era el ofrecimiento de una ayuda sin una consideración real de los intereses de los países del sur y la búsqueda de excelencia de los propios equipos de investigadores de los países del norte (Miguel y Ugartemendía, 2010; Bonfiglioli y Mari, 2000).

También Gaillard (1994) sostiene que uno de los principales problemas encontrados en la implementación de los programas de investigación colaborativa entre países más desarrollados y naciones menos avanzadas se relaciona con la asimetría de la colaboración y el dominio de los socios en el norte. Esto provocó que las colaboraciones hayan estado orientadas a las necesidades específicas de los países desarrollados.

Si bien desde los ochenta en adelante algunos organismos internacionales, como la UE y la Organización para la Cooperación y el Desarrollo Económicos (OCDE), llaman a un cambio que proponía una co-responsabilidad por parte de los países destinatarios de ayuda (Miguel y Ugartemendía, 2010, p. 3) esto no tuvo mayores efectos en la práctica de la cooperación internacional en CyT. Asimismo, Feld y Kreimer (2019, p. 784) describen una etapa que comienza en los noventa, "que se orientaría a generar estructuras de cooperación en las que el objetivo declarado es el beneficio mutuo" y la "retórica dominante" (Gaillard, 1994) apoyaba la búsqueda de beneficios para ambos lados. Pero bajo la apariencia de "libre acceso" a instrumentos/financiamiento europeos y "beneficio mutuo", permanecieron y se mantuvieron las asimetrías vinculadas con las posibilidades de la definición de las agendas y la generación y uso del conocimiento por parte de los países europeos (Bonfiglioli y Mari, 2000).

Es así que se constata que una crítica recurrente en los análisis de las formas tradicionales de la cooperación en CyT entre el norte y el sur (que comparten los análisis de la cooperación al desarrollo en su totalidad) es su carácter unidireccional. Es decir que se afirma que son ayudas que provienen de los países más desarrollados y se dirigen a los países más atrasados, sin una consideración acabada y completa de las necesidades reales de los países receptores. Este tipo de cooperación, al que se le reconocen algunos resultados positivos, no colaboraría con el desarrollo de las potencialidades de los sistemas productivos locales y tendría relativamente pocas consecuencias positivas sobre el desarrollo económico y social de los pueblos receptores de la ayuda internacional (Bonfiglioli y Mari, 2000, p. 184).

En esta línea de análisis, las transformaciones socio-políticas y económicas de principios del siglo XXI también provocaron cambios en el modo de valorar la cooperación científico tecnológica: la securitización de la agenda internacional ha provocado que la región latinoamericana pierda importancia estratégica para Europa (Sanahuja, 2005) sumado a la posterior crisis financiera internacional (se admite formalmente que tasas de crecimiento económico negativas en la UE como consecuencia de la crisis financiera y las medidas de austeridad introducidas por los Estados miembros llevaron a la reducción de la AOD) y a las variadas crisis endógenas de la UE (Alvarez y Cabeza, 2018). Todas estas situaciones vendrían a reforzar la característica de unidireccionalidad, mermando las posibilidades de los países receptores de intervenir en el diseño y puesta en práctica de la cooperación internacional en CyT.

Vale aclarar que desde 1984 en adelante, el apoyo de la UE a las actividades de investigación y desarrollo tecnológico se inscribió en iniciativas estratégicas denominadas "Programas Marco" (PM). Estos PM reflejan las principales iniciativas comunitarias de fomento y apoyo a la investigación y desarrollo en la UE, teniendo como principal objetivo la mejora de la competitividad a través de la financiación fundamentalmente de actividades de investigación, desarrollo tecnológico, demostración e innovación en régimen de colaboración transnacional entre empresas e instituciones de investigación pertenecientes tanto a los países de la UE y Estados Asociados, como de terceros países.

Los grupos de investigadores de América Latina comenzaron a participar desde el IV PM que se desarrolló en el período 1994-1998, cuando se establece por primera vez un programa específico de "Cooperación con Terceros Países y Organizaciones Internacionales" (INCO, 
International Cooperation) que incluyó la cooperación con países en desarrollo (INCO-DC, International Cooperation with Developing Countries) (Feld y Kreimer, 2019, p. 784; Gusmao, 2000, p. 135).

A partir del sexto y, sobre todo, del séptimo PM (período 2007-2013), se implementó una novedad en la estructura de la cooperación europea: el trabajo con países en desarrollo no presentaría diferencias significativas, en materia de instrumentos, respecto de la cooperación intra-europea o con países desarrollados. A partir de la organización de estos PM los terceros países pueden participar en pie de igualdad con los Estados miembros del subprograma "cooperación", y ya no lo hacen exclusivamente a través del INCO (Feld y Kreimer, 2019, p. $785)$.

Se debe remarcar que, dentro del período recién señalado, tuvo lugar la entrada en vigor del Tratado de Lisboa en diciembre de 2009, cuando se le otorga a la UE una voz única en cooperación internacional y se prioriza la coherencia en política exterior.

En el 2014 la terminología sufrió algunos cambios y ya no se menciona a los PM con un número. Así, específicamente en la cooperación en CyT, se acaba de implementar el programa "Horizonte 2020" que toma el período 2014-2020 y se sustenta sobre tres pilares y objetivos que pueden sintetizarse en estos términos: abordar los principales retos sociales, promover el liderazgo industrial en Europa y reforzar la excelencia de su base científica (San Segundo, 2020, p. 2).

Para dar continuidad al programa "Horizonte 2020" se presentó en febrero del presente año "Horizonte Europa" como un programa de financiación de la investigación y la innovación de 100.000 millones de euros que tendría lugar durante siete años (2021-2027) y que se propone como el mayor programa de investigación e innovación del mundo. El debate que dio lugar a este instrumento se produjo en medio de la pandemia y de la salida de Gran Bretaña de la UE, por lo que incluyó estas novedades y sus múltiples efectos. Este gran PM está estructurado sobre tres pilares denominados: Ciencia Excelente, retos globales y competitividad industrial europea, y Europa innovadora. Más de 140 países de fuera de la UE ya cuentan con convenios de participación para recibir fondos competitivos del programa marco (Campos, 2021).

Como se advierte, al estar regulada por "marcos" europeos, la modalidad de cooperación internacional en CyT se encuentra impregnada de los objetivos de los países miembros de la UE, y los terceros países se "amoldan", incorporándose al gran proyecto de los PM, ahora denominados con nombres más "atractivos". De esta observación crítica no se debe concluir que no se obtengan del trabajo en conjunto beneficios que fortalecen y enriquecen al sistema de conocimiento en CyT de la Argentina, pero claramente la estrategia global no está diseñada por las partes que pertenecen a la periferia, sino que la gran impulsora siempre es la UE. Y, en términos generales, no se evidencia que las políticas del norte busquen compatibilizar sus propósitos con los objetivos de los países menos adelantados.

Coincidimos con Rodríguez Medina (2014, p. 13) quien señala que esta direccionalidad única, que el autor advierte en la movilidad del conocimiento y en la divulgación de la ciencia, tiene sus consecuencias directas en la formación de las agendas, en la organización de los programas y en la tematización de los congresos y seminarios y en la organización de la movilidad académica.

Por otra parte, "a diferencia de la cooperación para el desarrollo, la política de cooperación internacional en ciencia y tecnología no se define en función de una obligación moral ('la ayuda al otro'), ni apela a una motivación ética global como 'luchar contra la pobreza', sino que responde al interés estatal de incrementar las propias capacidades del país, considerados recursos de poder" (Kern, 2008, p. 2). En estos casos entonces, los fines últimos están más claros y sincerados que en la cooperación internacional para el desarrollo. 


\section{La cooperación en CyT entre Argentina y la Unión Europea durante los gobiernos de Cristina Kirchner y Mauricio Macri}

Como se sostuvo en la introducción, se considera a la cooperación en CyT entre Argentina y la UE como una política nacional dentro del marco más general de la cooperación internacional y de la política en CyT a nivel nacional. En este marco, los datos de la cooperación en CyT de Argentina con el resto del mundo durante el período de gobierno de la presidenta Cristina Fernández de Kirchner (2007-2015) muestran que Europa y Estados Unidos fueron las principales contrapartes en la mayoría de los instrumentos de cooperación en CyT puestos en práctica.

Además, el Acuerdo de Implementación firmado entre el MINCYT y la Comisión Europea en marzo de 2015 convirtió a la Argentina en el primer país latinoamericano -y tercero en el mundo- en implementar una colaboración que permite a científicos de centros de investigación argentinos trabajar con investigadores europeos en el Consejo Europeo de Investigación (ERC, por sus siglas en inglés), organismo de financiación europeo consagrado a la investigación de excelencia (EsHorizonte2020, 2016).

Esta importancia otorgada por el gobierno de Cristina Fernández de Kirchner a los países del norte, "la cual se percibe como un vínculo de dependencia" (Demarchi, 2020, p. 88), se contradice con lo expresado a nivel discursivo por los representantes políticos que se mostraban a favor de un fortalecimiento de la cooperación internacional con América Latina junto con otras prácticas más horizontales. Durante esta etapa, si bien se revitalizaron las instituciones del Sistema Nacional de Ciencia y Tecnología y se actualizó parcialmente la deteriorada infraestructura otorgándole a la CyT un lugar de importancia para el país, los niveles de inversión global de nuestro país en I+D se mantuvieron en niveles por debajo de los competitivos a nivel internacional y la matriz productiva no llegó a modificarse significativamente (Stefani, 2018, p. 8).

Aunque América Latina ocupó un lugar destacado en el discurso público de los gobernantes, incluso en la posibilidad de generación de conocimiento en forma conjunta a nivel regional, en realidad se implementaron con mayor fuerza los programas y acciones con los países desarrollados. Es por ello que se sostiene que la cooperación en CyT con estos actores fue "uno de los pilares más importantes de la política de cooperación que llevó adelante la Dirección Nacional de Relaciones Internacionales del MINCYT" (Demarchi, 2018, p. 10). De modo tal que, durante este gobierno, ese Ministerio sostuvo una relación muy cercana y continua con la UE lo que se explica, entre otros motivos, por la existencia de una larga historia de relacionamiento que pesa fuerte en esa vinculación (Demarchi, 2018. p. 10; Kern, 2014, p. 5). Por parte de la UE es importante tener en cuenta que la región ha recurrentemente intentado exportar su modelo de integración a otras partes del mundo y con ninguna región lo ha hecho de manera más regular y persistente que con ALC, a quien la UE considera un aliado político privilegiado y, al mismo tiempo, una plataforma para extender su propio sistema centrado en la integración regional como fórmula para alcanzar la paz, la democracia y el desarrollo (Alvarez Macías, 2010, p. 4; Gratius, 2017, p. 64).

La necesidad de financiamiento y de búsqueda de fondos se tradujo en una limitación al tomar decisiones basadas en las propias necesidades, que respondan de la mejor manera posible a la estrategia de desarrollo local. Si bien durante el gobierno que se analiza se concibió con amplio consenso un plan estratégico de mediano plazo, el Plan Argentina Innovadora 2020, el mismo se ejecutó parcialmente (Stefani, 2018, p. 4). Estas falencias hacen que pierda fuerzas el supuesto defendido por el gobierno kirchnerista respecto a la importancia de la CyT como herramienta para alcanzar la autonomía nacional y mejorar la calidad de vida de sus habitantes.

Al asumir el nuevo gobierno de Mauricio Macri en diciembre del 2015, el Ministerio de Ciencia, Tecnología e Innovación Productiva fue el único ministerio que no sufrió modificaciones en su jefatura. Por esta decisión, Lino Barañao se mantuvo en su cargo, lo que puede ser leído como un reconocimiento a la política en CyT implementada por el gobierno de Cristina Fernández de Kirchner (Demarchi, 2020, p. 89). Aun así, en el 2018 este Ministerio (creado en el 2007 por la gestión anterior) pierde su rango y se convierte en Secretaría, pasando a depender del Ministerio 
de Educación (renombrado Educación, Cultura, Ciencia y Tecnología). Consideramos que en la nueva estructura burocrática la cooperación internacional perdió jerarquía dado que, en Argentina, cuando un Ministerio se transforma en Secretaría delega autonomía, tiene menos peso simbólico y pueden surgir más trabas presupuestarias para la implementación de políticas. De esta manera, existe una congruencia entre las estructuras estatales existentes y los objetivos del gobierno. "La burocracia es la expresión material del Estado y el brazo que ejecuta sus políticas" (Oslak, 2006, p. 3), de ahí la importancia de la modificación de la estructura del Estado y del pasaje de Ministerio a Secretaría. Esta transformación burocrática negativa tiene como consecuencia, además, el desaprovechamiento de la buena reputación lograda:

Más allá de que el Ministerio no pudo resolver el viejo problema que marcó desde sus comienzos al funcionamiento del Sistema Nacional de Ciencia y Tecnología argentino asociado a la ausencia de articulación entre sus organismos, representó un modelo burocrático que adquirió legitimidad, que se caracterizó por la asignación transparente de los fondos, que logró conformar un plantel profesional, que le dio otro status al área y otro tipo de interlocución externa. De ahí el fuerte impacto negativo que implicó su abolición, no sólo para la comunidad científica sino para la sociedad en general (Demarchi, 2020, p. 90).

En forma paralela con estas modificaciones burocráticas en el organigrama ministerial, durante la gestión de gobierno de Mauricio Macri, el presupuesto nacional destinado a la ciencia pasó del 0,35 al 0,23\% del PBI entre 2015 y 2018 (Taranto, 2019). En el 2017 se redujo en un $60 \%$ la incorporación de nuevos científicos en el CONICET, revirtiendo la tendencia expansiva registrada consecutivamente desde 2004. Así, la cantidad de becas que otorgó el organismo para ser implementadas a partir del 2018 se redujo a la mitad respecto de $2015^{4}$.

Es así que la inversión pública destinada a investigación científica y desarrollo tecnológico "se ha ido reduciendo de manera sistemática en valor real, se han desfinanciado y discontinuado programas de investigación y de formación de recursos humanos, los incipientes ecosistemas tecnológico productivos como el nuclear y el satelital fueron desensamblados, y no se han implementado políticas que impulsen la inversión privada I+D de manera significativa" (Stefani, 2018, p. 9).

Al igual que lo sucedido durante el gobierno de Cristina Fernández de Kirchner, nuevamente fue clara la distancia entre lo declarado a nivel discursivo y lo que luego tiene lugar a través de acciones y decisiones vinculadas a la política de CyT a nivel nacional. Durante el gobierno de Mauricio Macri "a pesar de lo explicitado en los distintos documentos y declaraciones oficiales...se emprendieron acciones aisladas, espasmódicas, concentradas sobre todo en la participación en eventos internacionales, y sin ningún tipo de articulación entre el MINCYT y el MREC” (Demarchi, 2020, p. 90).

Por último, ninguna de las dos gestiones pudo resolver el problema estructural relacionado con la fragmentación institucional del Sistema Nacional de Ciencia y Tecnología ${ }^{5}$. Esto dificultó la construcción de una agenda de cooperación estratégica, unificada, que promoviera el cumplimiento de los objetivos e intereses nacionales. A ello hay que sumarle la

\footnotetext{
${ }^{4}$ El Gobierno ratificó el recorte en el Conicet: habrá menos becas en 2018. 17de febrero de 2017. Minuto Uno. Recuperado de: https://www.minutouno.com/politica/conicet/el-gobierno-ratifico-el-recorte-elhabra-menos-becas-2018-n1537305

5 Las instituciones que realizan investigación científica y desarrollos tecnológicos en la Argentina (CONICET, CONAE, CNEA, INTI, INTA, Universidades Nacionales, entre otras) no logran adquirir cohesión y proponer un trabajo articulado. Este conjunto de instituciones no tiene las particularidades de un Sistema Nacional de Ciencia y Tecnología ya que carece de coordinación interna y se encuentra mayormente desconectado de aquellos sectores con los que debería interactuar (aparato productivo, sistemas de salud y educación, áreas de energía y recursos naturales, defensa, etc.). Esta fragmentación institucional e intersectorial es característica de los países dependientes y se acompaña con la ausencia de un proyecto nacional sustentable. Para profundizar sobre las causas históricas de esta desconexión entre la "oferta de conocimientos" por parte de los centros de investigación y las "demandas de innovación" de parte de las empresas puede verse la investigación de Bonfiglioli y Mari (2000).
} 
desarticulación entre el MINCYT y el MRECIC; y el hecho de que cada organismo tenía su propia agenda externa.

La cooperación en CyT de Argentina con la UE durante los últimos años del gobierno de Mauricio Macri seguía siendo fuerte: un indicador importante es la participación del país en el programa Acciones Marie Skłodowska-Curie (MSCA), que fomenta la movilidad de los investigadores en todos los niveles: desde que comenzó el programa "Horizonte 2020" en el 2014 hasta junio del 2018, Argentina fue el país con mayor participación en la región de Latinoamérica, con 77 organizaciones que formaron parte de 56 proyectos, involucrando a 307 investigadores argentinos y 168 investigadores extranjeros en instituciones argentinas (Estado Argentino, 2018).

Los temas que siguieron vigentes durante este gobierno se pueden rastrear en los actos y conferencias que tuvieron lugar durante celebración de los 20 años de cooperación conjunta. Para esta ocasión, se organizaron talleres, reuniones y conferencias que tuvieron lugar en junio de 2019 (Estado Argentino, 2019).

Se delinean en el Comunicado Conjunto Argentina-UE (2019) cuatro líneas temáticas prioritarias:

1. El ámbito de la investigación marina, donde la actividad se desarrolla en el marco del Acuerdo Administrativo firmado en el 2017 que facilita la participación activa de científicos de ambas regiones en una serie de iniciativas clave que tienen como objetivo estudiar los océanos y que son muy relevantes para la comprensión del cambio climático y ecosistemas marinos.

2. Bioeconomía: se reconoce la importancia del "Foro Internacional de Bioeconomía" como espacio para la cooperación internacional regular y estratégica y se declara la intención de fortalecer los grupos de trabajo sobre microbioma y bosque.

3. Salud: previo a la pandemia, se demostró un evidente interés en los proyectos conjuntos sobre cáncer, la futura cooperación argentina bajo la Alianza Global para Enfermedades Crónicas y la colaboración con Global Research para Enfermedades Infecciosas, entre otros programas del área de Salud.

4. Infraestructuras de investigación (centros que ofrecen recursos y servicios a las comunidades de investigación para que puedan ejercer su labor e impulsar la innovación): se trabaja en pos del objetivo de abrir el acceso transfronterizo a las infraestructuras de investigación en las dos regiones (UE y ALC) y compartir las mejores prácticas.

Además, Argentina fue el primer país latinoamericano en unirse a las Noches de Investigadores Europeos, un evento de divulgación científica que acerca a los expertos con el público en general, con actividades basadas en el Centro Cultural de la Ciencia, en simultáneo con más de 370 ciudades europeas. En el mencionado Comunicado Conjunto, la UE también acogió con satisfacción la participación de Argentina en Erasmus+ y alentó a una mayor participación en, por ejemplo, proyectos de creación de capacidad en el campo de la educación superior.

Con respecto al ERC, un buen número de investigadores argentinos han pasado períodos en la institución, basada en la excelencia de sus proyectos, colocando al país en la cima entre los países del ALC.

Complementariamente a estos escenarios, la UE anunciaba que desde 2019 las subvenciones Synergy otorgadas por el ERC, estarían abiertas a los principales investigadores con sede fuera de la UE y alentaron a los investigadores argentinos para aprovechar esta oportunidad.

\section{El gobierno de Alberto Fernández. Algunas reflexiones en la coyuntura de la pandemia}

Con la llegada del nuevo gobierno de Alberto Fernández en diciembre del 2019, y entre las primeras acciones vinculadas al área de CyT, se decide la devolución de rango de Ministerio al área de Ciencia y Tecnología, poniendo a cargo del mismo al bioquímico Roberto Salvarezza. En principio, esta decisión permitiría recuperar independencia en las decisiones dentro de la 
estructura gubernamental y un mejor manejo del presupuesto para el área de la Cooperación Internacional en CyT.

Haciendo referencia nuevamente al contexto político nacional en el que se da la cooperación en CyT entre Argentina y la UE, se puede marcar un avance en la legislación argentina: la sanción de la Ley $\mathrm{N}^{\mathrm{o}} 27.614$ de Financiamiento del Sistema Nacional de Ciencia, Tecnología e Innovación ${ }^{6}$. Esta ley representa un logro en la histórica lucha de la comunidad científica para que los recursos destinados al área de CyT tengan una participación del $1 \%$ del Producto Bruto Interno (PBI) del país. La legislación, que fue aprobada en el Senado con el apoyo de todas las fuerzas políticas, dispone que el Estado Nacional deberá ir aumentando el presupuesto destinado a la función CyT (de un actual 0,28\% del PBI) hasta llegar al 1\% del PBI en el 2032. Entre las argumentaciones para impulsar la ley se incluyó la nueva situación de emergencia por la pandemia. Así, la directora del CONICET, Ana María Franchi afirmó:

La pandemia demostró la importancia de contar con un desarrollo tecnológico nacional. Cambió el escenario hacia afuera y hacia adentro. Hacia afuera porque demostramos que nosotros estábamos para algo, que había necesidad de una comunidad científica tecnológica en el país. Y hacia adentro se demostró que teníamos una formación, que teníamos la tecnología para poder movernos de nuestros temas específicos de investigación y hacer cosas para ayudar a resolver problemas. Fue una enseñanza (Cafferata, 2021).

Actualmente y en un marco general, la cooperación de la UE con Argentina se estructura a través de la asistencia técnica, el intercambio de experiencias y la creación de redes. Las prioridades de la cooperación europea en la totalidad del escenario mundial están sostenidas en el Pacto Verde Europeo para lograr una economía sostenible (también llamado Libro Verde) lanzado en diciembre de 2019, que funciona como hoja de ruta para lograr una economía sostenible. Los grandes ejes de trabajo que se declaran como prioritarios en la colaboración entre la UE y Argentina son: Cambio climático, medioambiente, y energía; Cohesión social; Igualdad de género; Cooperación universitaria y científica, Justicia y seguridad, Cooperación económica, comercial e inversión y, por último, Derechos humanos y fortalecimiento de la sociedad civil (Delegación de la Unión Europea en la Argentina, 2020).

Durante los días 1 y 2 de diciembre del 2020 se llevó a cabo la Undécima Comisión Mixta UE-Argentina de manera virtual a causa de la situación de salud pública generada por la pandemia. En esta ocasión la cooperación internacional tuvo sus menciones y se renovaron, siempre a nivel discursivo, los esfuerzos comunes para otorgarle a la misma un sentido estratégico (Delegación de la Unión Europea en la Argentina, 2020).

Asimismo, durante el 2020 la Delegación de la UE en la Argentina abrió una convocatoria para una línea de proyectos, con un presupuesto de 6,15 millones de euros, para fortalecer la capacidad de la sociedad civil de la Argentina en temas de cuestiones sociales y desarrollo sustentable. Además, en el mismo proyecto se incluyó una convocatoria sobre temas indígenas, derechos sociales y los accesos a educación y salud, a los servicios básicos, con una mirada sobre la situación socio-sanitaria y económica actual. El jurado que evaluará los proyectos estará integrado por funcionarios de la $\mathrm{UE}^{7}$.

De cualquier manera, desde un enfoque general, se sigue observando que, más allá de los proyectos, investigadores involucrados, instituciones financiadas y otras herramientas de cooperación importantes, predomina la unidireccionalidad en el diseño de los objetivos. Habitualmente, siempre es la UE quien diseña las estrategias y luego "invita" (selecciona, evalúa

\footnotetext{
${ }^{6}$ Ley de Financiamiento del Sistema Nacional de Ciencia, Tecnología e Innovación, Ley 27.614. Boletín Oficial de la República Argentina, 12 de marzo de 2021. Recuperado de: https://www.boletinoficial.gob.ar/detalleAviso/primera/241782/20210312

${ }^{7}$ La UE destina 6,15 millones de euros en subvenciones para proyectos de asociaciones civiles argentinas, 16 de junio de 2020, Telam. Recuperado de: https://www.telam.com.ar/notas/202006/475560-unioneuropea-dinero-proyectos-asociaciones-civiles-argentina.html
} 
y financia) a participar a científicos argentinos. Por supuesto que los beneficios existen, pero son relativizados por esta falta de participación en la toma de decisiones en el momento de diseñar los grandes programas. Entre los resultados positivos, verificados en varios países de América Latina, está la constitución de grupos científicos que "han alcanzado en no pocos casos niveles de excelencia que los ubican a la altura de los mejores centros internacionales" (Bonfiglioli y Mari, 2000, p. 184). Vale aclarar, entonces, que el sistema de cooperación bajo análisis es extremadamente complejo y amplio como para cerrar definitivamente esta evaluación. Se coincide con Gusmao (2000, p. 160) cuando afirma, en un nivel latinoamericano pero aplicable al nacional, que "la complejidad de todo el sistema, la diversidad de factores y de actores movilizados, plantean de por sí un manifiesto problema de evaluación, luego de 'medidos' los efectos y los verdaderos impactos de la política en el sector".

En un similar intento de valorar la cooperación en CyT con la UE, Feld y Kreimer (2019, p. 785) sostienen que, incluyendo la mirada del paradigma limitado o "políticas para las ciencias" o del paradigma ampliado o "políticas a través de las ciencias", en ambos casos las motivaciones europeas para la cooperación científica se basan en una estrategia destinada a superar las desventajas de la región con respecto a otros competidores globales, como Estados Unidos o Japón. Las limitaciones para nuestro país que implican las intenciones que sustentan a este tipo de cooperación se potencializan y se refuerzan en el nuevo escenario mundial surgido a partir de la pandemia de Covid-19 en los primeros meses del 2020. Esto es así porque es de esperar un reforzamiento de los intentos por parte de la UE de utilizar la cooperación en CyT exclusivamente con objetivos propios.

Además, junto con la relativa pérdida de hegemonía norteamericana y el interrogante abierto sobre si la República Popular China podrá hacerse cargo de ese liderazgo, se podría adelantar que la clásica orientación norte-sur de la cooperación internacional ha mostrado señales de cambio en el contexto de la pandemia (Morasso, 2020). Mucho antes se delineaba ya con fuerza un cambio en la percepción regional, solicitando más simetría y proponiendo a los países del sur nuevas opciones de cooperación sur-sur y cooperación triangular (Morazán, Sanahuja y Ayllón, 2011, p. 15).

En la coyuntura global provocada por la pandemia, China ha estado en primera línea con suministros médicos y ayuda humanitaria, posicionándose como un cooperante mundial. En plena crisis, las instituciones multilaterales de alcance global han sido llamadas a un protagonismo, aunque esta intervención en algunas ocasiones se vio alterada por la falta de transparencia y eficacia. Durante el 2020 la ausencia de consenso limitó el accionar de Naciones Unidas y de su Secretario General.

Según Hirst y Malacalza (2020, p. 6), entre los efectos de la situación de emergencia sanitaria, con proyecciones económicas muy negativas, se encuentra la "probable implosión" de la Agenda 2030, cuyos objetivos de reducción de la pobreza serán más difíciles de alcanzar. Según estos autores, en Europa el avance del coronavirus sacó a relucir cuatro tendencias que ya venían manifestándose: la crisis de la UE como modelo de la integración regional, región de paz, bienestar y democracia; la emergencia de los nacionalismos de extrema derecha y el giro autoritario en algunos países del Este; la división entre el norte y el sur europeos; y, finalmente, la creciente influencia económica y tecnológica de China en la región, junto con las señales por parte de Estados Unidos, durante el gobierno de Trump, de relativizar su apoyo a la Organización del Tratado del Atlántico Norte (OTAN) (Hirst y Malacalza, 2020, p. 8). Esto último refiere a la decisión de retirar tropas estadounidenses de Alemania. En síntesis, la pandemia y sus efectos, la exacerbación de la disputa entre China y Estados Unidos, junto con otras tendencias hacia el interior del grupo, profundizaron divisiones que ya existían en la UE.

\footnotetext{
${ }^{8}$ Estas tipologías refieren a las "políticas para la ciencia", que se proponen como objetivos mejorar la calidad, el alcance y la masa crítica de la investigación, en definitiva que los resultados nutran al propio sistema científico; y a las "políticas a través de las ciencias", que intentan mejorar la competitividad, enfrentar desafíos sociales globales y respaldar a los países menos desarrollados en la construcción de capacidades en CyT, o sea que los resultados se extiendan a otros ámbitos más amplios.
} 


\section{Conclusiones}

Una primera conclusión, luego del recorrido analítico realizado, lleva a afirmar que Argentina mantiene su fuerte presencia en todos los programas propuestos dentro la cooperación científico técnica con la UE. Se encuentra en los primeros lugares entre los países de América Latina y los científicos y tecnólogos argentinos participan con una alta tasa de éxito en las convocatorias internacionales que suponen trabajo colaborativo y que reciben financiamiento de la Comisión Europea.

Como contrapartida de estas constataciones, se afirma que la cooperación en CyT entre Argentina y la UE está apuntalada en el modelo tradicional de cooperación norte-sur o, para ser más específicos, entre países centrales y países periféricos o semi periféricos, con todas las falencias señaladas: marcos de cooperación dados desde el norte hacia el sur, poca o nula posibilidad de intervención en el diseño de los modelos de cooperación, agendas cerradas con objetivos foráneos, entre otras limitaciones.

La UE se posiciona como el proveedor de fondos que decide a quién darlos y para qué, y además evalúa si se han usado bien o mal. La capacidad de procesamiento de la información sigue centralizada en los países europeos. Este esquema de cooperación tradicional, que proviene del formato de cooperación norte-sur, es uno de los más estáticos y difíciles de adaptar, por lo que los cambios globales provocados por la emergencia sanitaria y la pandemia parecen no haber provocado aún consecuencias o derivaciones visibles.

Pero, pensando en las transformaciones globales que están teniendo lugar, es inevitable esperar un impacto en la cooperación en CyT a nivel mundial. Atendiendo además que la actual situación es producto de una crisis sanitaria, tan vinculada a los ámbitos de la ciencia, la investigación y la tecnología, es de esperar impactos fuertes. En el régimen internacional de ayuda y cooperación en su totalidad se están dando transformaciones actualmente en curso, y en el escenario mundial han surgido nuevos liderazgos internacionales, alternativos al de Estados Unidos y a Europa. Con cambios en las hegemonías y poderes más difusos se transformarían las narrativas que sustentan la cooperación internacional y el rol que juega la cooperación científico y técnica.

¿Cómo se articularán, con estas transformaciones, la cooperación internacional desde la UE hacia la Argentina? ¿Cómo compartirán escenarios la "nueva arquitectura de la cooperación internacional" con los viejos moldes o antiguas modalidades de cooperación?

En una coyuntura compartida de caída de la actividad económica provocada por una crisis sanitaria sin precedentes, se observa que las potencialidades para hacer frente a la emergencia son muy diferentes y las capacidades de respuestas contracíclicas también. Entre otras cuestiones, se observa que la UE puede hacer uso de emisión de moneda y de endeudamiento como medidas para enfrentar al Covid-19 mientas que en los países de América Latina las posibilidades son muy diferentes. Así, las perspectivas a mediano plazo estarán relacionadas, una vez más, con los modelos de desarrollo propios. En este contexto, se deberá intentar con la cooperación internacional mejorar las políticas públicas, promover la lucha contra el cambio climático y reforzar la gobernanza global. Esta cooperación internacional sólo será posible con la conformación de un nuevo multilateralismo que se estaría gestando. Pero las respuestas de este tipo tardan en llegar. En la espera, se profundiza la desigualdad. La gravedad de la situación se completa con el pronóstico de repeticiones de pandemias o crisis sanitarias en un futuro próximo que, para ser enfrentadas, requieren de acciones globales.

La irrupción de la pandemia de Covid-19 trajo cambios visibles e invisibles, que se articulan con situaciones de desigualdad pre existentes, generando nuevas injusticias, con implicancias políticas, sociales y culturales, entre otras. No será posible, o no debería ser deseable, pensar en un "regreso a la normalidad" sin replantearse muchas de las desigualdades que precedieron, y en cierta forma, explican esta crisis pandémica. Entre los replanteos sigue la necesidad de implementar una cooperación que tenga en cuenta los intereses de los países 
receptores, que los programas se basen en sus demandas reales y los resultados positivos sean equilibrados para ambas partes.

Por otro lado, es simple prever que, en los ámbitos de cooperación internacional, y específicamente en la cooperación en CyT, será difícil superar el egocentrismo y el cierre hacia dentro que está mostrando la UE en estos tiempos difíciles.

\section{Bibliografía citada}

Alvarez Macías, M. V. (2010). "Las relaciones de la Unión Europea y América Latina a la luz del Tratado de Lisboa: Algunos escenarios futuros posibles". Revista Integración y Cooperación Internacional (3), pp. 2-6. Recuperado de https://rephip.unr.edu.ar/handle/2133/6398

Alvarez, M. V. y Cabeza, M. (2018). La Unión Europea en contexto de crisis: dimensiones y claves para su análisis. E-book, Rosario: UNR Editora. Recuperado de https://rephip.unr.edu.ar/bitstream/handle/2133/14316/LA\%20UNI\%C3\%93N\%20EUR OPEA\%20EN\%20CONTEXTO\%20DE\%20CRISIS.pdf?sequence $=3 \&$ is Allowed $=\mathrm{y}$

Ámbito Internacional (s.f.). Argentina-Unión Europea celebran 20 años de cooperación. Recuperado de http://ambitointernacional.com/argentina-union-europea-celebran-20anos-de-cooperacion/

Bonfiglioli, A. y Mari, E. (2000). La cooperación científico-tecnológica entre la Unión Europea y América Latina: el actual contexto internacional y el Programa Marco de la Unión Europea. Revista REDES, Buenos Aires, Universidad Nacional de Quilmes, 7(15), pp. 183-208

Cafferata, M. (2021). Ley de Financiamiento para Ciencia y Técnica: El gran triunfo del uno por ciento, en Página 12, 26 de febrero. Recuperado de https://www.pagina12.com.ar/326200-ley-de-financiamiento-para-ciencia-y-tecnica-elgran-triunfo

Campos. A. (2021). La UE presenta Horizonte Europa, el mayor programa de investigación e innovación del mundo. Observatorio de Cibermedios, Universitat Pompeu Fabra. 8 de febrero. Recuperado de https://observatoriocibermedios.upf.edu/horizonte-europaciencia

Caria, S. (2020). Las agendas de cooperación internacional como instrumentos de promoción comercial: perspectivas para América Latina. En S. Caria e I. Giunta (coordinadoras). Pasado y presente de la cooperación internacional: una perspectiva crítica desde las teorías del sistema mundo (pp. 263- 292). $1^{\text {a }}$ Ed. Quito: Editorial IAEN

Delegación de la Unión Europea en la Argentina (2020). $11^{a}$ Comisión Mixta UE-Argentina: Comunicado de prensa conjunto, 3 de diciembre. Recuperado de https://eeas.europa.eu/delegations/argentina/89874/11\%C2\%AA-comisi\%C3\%B3nmixta-ue-argentina-comunicado-de-prensa-conjunto_es

Demarchi, P. (2018). La cooperación internacional en ciencia y tecnología argentina. Revista Integración y Cooperación Internacional, (26), pp. 5-14. https://doi.org/10.35305/revistamici.v0i26.28

Demarchi, P. (2020). Cooperación internacional en Ciencia y Tecnología: cambios y continuidades en los gobiernos de Cristina Fernández (2007-2015) y Mauricio Macri (2015-2018). En N. Ceppi y M.E. Lorenzini (editoras). Zooms sudamericanos: agendas, 
vínculos externos y desafíos en el Siglo XXI (pp. 80-94). Recuperado de https://www.iaen.edu.ec/wp-content/uploads/2020/04/Zoomssudamericanos_agendas_v\%C3\%ADnculos-y-desaf\%C3\%ADos-en-el-siglo-XXI.pdf

Denk, A. (2016). Sustainable Development Goals-An (Alternative) Future Scenario. Transcience, 7 (1), pp. 47-50

EsHorizonte2020 (2016). El acuerdo Argentina-ERC da sus primeros resultados. Portal español del Programa Marco de Investigación e Innovación de la Unión Europea. 11 de julio. Recuperado de https://eshorizonte2020.es/ciencia-excelente/consejo-europeo-deinvestigacion-erc/noticias/el-acuerdo-argentina-erc-da-sus-primeros-resultados

Estado Argentino (2018). Trece organizaciones argentinas fueron seleccionadas para las acciones RISE 2018. 11 de julio. Recuperado de https://www.argentina.gob.ar/noticias/trece-organizaciones-argentinas-fueronseleccionadas-para-las-acciones-rise-2018

Estado Argentino (2019). 20 años de cooperación entre Argentina y la Unión Europea en Ciencia y Tecnología. 1 de junio. Recuperado de https://www.argentina.gob.ar/noticias/20-anosde-cooperacion-entre-argentina-y-la-union-europea-en-ciencia-y-tecnologia

Feld, A. y Kreimer, P. (2019). ¿Cosmopolitismo o subordinación? La participación de científicos latinoamericanos en programas europeos: motivaciones y dinámicas analizadas desde el punto de vista de los líderes europeos. História, Ciências, Saúde - Manguinhos, Rio de Janeiro, v.26, n.3, jul.-set., pp. 779-799

Gaillard, J. F. (1994). North-South Research Partnership: Is collaboration possible between Unequal Partners. Knowledge and Policy: the international journal of knowledge transfer and utilization, Summer, 7(2), pp. 31-63

Grabendorff, W. (2014). Realidad y ficción en las relaciones entre CELAC y la Unión Europea. En A. Bonilla y G. Jaramillo (Editores). La CELAC en el escenario contemporáneo de América Latina y el Caribe (pp. 175-192), San José, Costa Rica: FLACSO SG

Gratius, S. (2017). Europa-América Latina: retos regionales y globales compartidos. Nueva Sociedad, 270, pp. 119-131

Gusmao, R. (2000). La implicación de los países latinoamericanos en los programas europeos de cooperación CyT con terceros países. Revista REDES, Buenos Aires, Universidad Nacional de Quilmes, 7(16), pp. 131-163

Hirst, M., y Malacalza, B. (2020). ¿Podrá reinventarse el multilateralismo? El orden internacional y el coronavirus. Nueva Sociedad, 287, pp. 35-48

Joint Communiqué. XI Joint Steering Committee Meeting of the Bilateral Agreement on Science and Technology between the European Union and Argentina. June $7^{\text {th }}$. Recuperado de https://www.scienceguide.n1/wp-content/uploads/2019/07/ec_rtd_jc-11th-jscm-euar_062019.pdf

Kern, A. (2008). Intereses, identidades, discursos e interacciones en la cooperación internacional en ciencia y tecnología. Observaciones de un estudio de caso entre Argentina y Alemania en el campo de las Tecnologías Informáticas. Miríada, Vol. 1, № 2, pp. 19-45

Kern, A. (2014). La agenda científica y tecnológica en los regionalismos de América Latina. Presentación en la Conferencia Internacional Conjunta FLACSO-ISA, 23 al 25 de julio. Recuperado de http://web.isanet.org/Web/Conferences/FLACSOISA\%20BuenosAires\%202014/Archive/9705df48-1e10-4e32-95fe-f46fc48f5992.pdf

Miguel, S. y Ugartemendía, V. (2010). Una mirada multinivel de la cooperación científica: La colaboración de Argentina con la Unión Europea en los niveles formal, instrumental y de las prácticas [en línea]. V Seminario Internacional sobre Estudios Cuantitativos y Cualitativos de la Ciencia y la Tecnología "Prof. Gilberto Sotolongo Aguilar", 19 al 23 
de abril, La Habana, Cuba. Recuperado de http://www.memoria.fahce.unlp.edu.ar/trab_eventos/ev.3942/ev.3942.pdf

Morasso, C. (2020). La cooperación internacional en tiempos del Covid-19. Un mapeo inicial. Síntesis Mundial, 8 de abril. Recuperado de: https://sintesismundial.wordpress.com/2020/04/08/la-cooperacion-internacional-entiempos-del-covid-19-un-mapeo-inicial/

Morazán, P., Sanahuja, J.A. y Ayllón, B. (2011). Una nueva política de cooperación para el desarrollo de la Unión Europea con América Latina. Parlamento Europeo, Dirección General de Políticas Exteriores de la Unión, Dirección B, Departamento Temático, EXPO/B/DEVE/FWC/2009-01/Lot5/18, diciembre

Müller, J. (2017). La era de la Perplejidad: Repensar el mundo que conocíamos. El auge ¿imparable? del populismo. Madrid: BBVA, OpenMind, Penguin Random House Grupo Editorial

Oslak, O. (2006). "Burocracia Estatal: Política y Políticas Públicas”. En Revista PostData, N 11

Parlamento Europeo (2020). Respuesta del Alto representante y vicepresidente Borrell en nombre de la Comisión Europea, Respuestas Parlamentarias, 15 de mayo. Recuperado de https://www.europarl.europa.eu/doceo/document/E-9-2020-000617-ASW ES.html

Pattacini, V. (2013). Los Países de Renta Media: Una lectura política y debates recientes sobre su rol y su desempeño en la Cooperación Sur-Sur del espacio iberoamericano. En M. Colacrai (comp). La Cooperación Internacional desde la visión de los PRM: discusiones conceptuales, diseños de políticas y prácticas sudamericanas (pp. 15-36). Rosario: UNR Editora. E-book. Recuperado de https://issuu.com/colacrai/docs/la cooperacion internacional

Rodríguez Medina, L. (2014). Construyendo periferia: un microanálisis de objetos subordinantes como tecnologías epistémicas. Sociológica, 29(83), pp. 9-46

San Segundo, R. (2020). Blog "De Madrid a Europa". Recuperado de http://www.madrimasd.org/blogs/demadridaeuropa/2020/11/12/133128

Sanahuja, J. A. (2005). Seguridad, desarrollo y lucha contra la pobreza tras el 11-S: los Objetivos del Milenio y la "securitización" de la ayuda. Documentación Social. pp. 25-41

Sanahuja, J. A. (2012). Las cuatro crisis de la Unión Europea. En M. Mesa (coord.). Cambio de ciclo: crisis, resistencias y respuestas globales. Anuario 2012-2013 (51-84). Madrid: CEIPAZ

Sanahuja, J.A. y Tezanos Vázquez, S. (2017). Del milenio a la sostenibilidad: retos y perspectivas de la Agenda 2030 para el desarrollo sostenible. Política y Sociedad, 54(2), pp. 533-555

Stefani, F. (2018). Rol actual y futuro de la ciencia en la innovación industrial y el crecimiento económico en Argentina. Centro de Investigaciones en Bionanociencias (CIBION). Recuperado de https://www.biologia.org.ar/wp-content/uploads/2018/10/Stefani2018_Rol-actual-y-futuro-de-la-ciencia_en-la-innovacion-industrial-en-Argentina.pdf

Taranto, P. (2019). Un cientificidio que pone en riesgo el futuro. Tiempo Argentino, 1 de septiembre. Recuperado de https://www.tiempoar.com.ar/informacion-general/uncientificidio-que-pone-en-riesgo-el-futuro/ 